\title{
Bowel injury in children: high-risk mechanisms and CT findings
}

\author{
George A. Taylor
}

(C) Springer-Verlag 2011

\section{Introduction}

Bowel and mesenteric injuries are, fortunately, uncommon complications of blunt abdominal trauma, occurring in $6-16 \%$ of injured children and adults. However, unlike injury to solid organs in which operative intervention is rarely necessary, injury to hollow viscera often requires early surgical repair. Delayed diagnosis can result in bowel ischemia, peritonitis and sepsis. Imaging plays a key role in the early and accurate identification of these lesions. This review will focus on high-risk mechanisms of injury and physical findings, as well as the frequency, sensitivity and specificity of CT findings of significant bowel and mesenteric injuries.

\section{High-risk mechanisms of injury}

The most common mechanisms of injury associated with bowel and mesenteric injury are (in descending order of frequency) motor vehicle crash $>$ handlebar injury $>$ nonaccidental trauma $>$ fall from height. These mechanisms cause bowel injury as the result of direct force on the gastrointestinal tract leading to a crush injury; rapid deceleration producing a shearing force between fixed and mobile portions of bowel; and a sharp increase in intraluminal pressure resulting in rupture of the gut. The presence of linear abdominal wall bruising caused by a

Disclaimer Dr. Taylor has no financial interests, investigational or off-label uses to disclose.

G. A. Taylor $(\bowtie)$

Children's Hospital Boston,

Boston, MA 02115, USA

e-mail: george.taylor@childrens.harvard.edu seat belt and acute hyper-flexion (Chance) fractures of the lumbar spine are the only physical findings that have been found to have a significant and high association with bowel/mesenteric injury.

\section{CT findings of bowel and mesenteric injury}

The main challenge for the radiologist is to distinguish between injuries that need surgical correction and those that do not. Findings specific to bowel injury include the presence of extraluminal gas, extravasation of oral contrast agent, and bowel wall discontinuity. Extraluminal gas must be evaluated with the mechanism of injury, associated thoracic injuries and recent treatment in mind. Extraluminal gas is present in $20-30 \%$ of children with bowel injury and is highly specific for bowel injury. Unfortunately, it has a relatively low (20-30\%) sensitivity for significant bowel injury. In our experience, pneumoperitoneum as a falsepositive finding is uncommon but is sometimes seen as the result of penetrating abdominal injury, large tension pneumothorax, genital injury in girls, or after diagnostic peritoneal lavage. In the supine patient, extraluminal gas tends to accumulate at the convexity of the abdominal wall, in the porta hepatis, mesenteric and portal veins. Injury to the retroperitoneal duodenum might result in localized bubbles of gas immediately adjacent to a thickened and distorted duodenum and pancreas. Careful evaluation of this area is necessary to distinguish between duodenal hematoma with and without perforation. Identification of extraluminal oral contrast agent is also very specific for bowel rupture. However, since the majority of pediatric trauma centers in North America do not routinely use oral contrast agents, this finding is rare $(<2 \%)$ and must be distinguished from extravasated urinary contrast agent 
arising from an intraperitoneal bladder perforation. Finally, identification of bowel discontinuity has been reported in injured adults but is very rare in children.

Several findings are commonly encountered in injured patients but are less specific to bowel injury. These include the presence of focal bowel wall thickening $(13 \%)$ and mesenteric fluid or stranding (31\%). Because of the general lack of pre-existing conditions in children compared to adults, it is unclear whether these findings are more specific in pediatric practice.

Highly specific findings for significant mesenteric injury are those related to vascular injury and include mesenteric vascular beading, abrupt termination of mesenteric vessels, and mesenteric vascular extravasation. However, these findings have been primarily reported in adults with serious mesenteric injury. They are rare findings in children, and their specificity/sensitivity is not known. Mesenteric pseudoaneurysm has been reported in children (3\%) and is usually treated by catheter occlusion. Abnormalities that are less specific for mesenteric injury requiring operative intervention are mesenteric stranding (31\%) and hematoma (7\%). These might be present as isolated findings or as an indicator of more serious underlying vascular disruption to the mesentery.

One CT feature that is common to both bowel and mesenteric injuries is the presence of unexplained peritoneal fluid. Defined as hemoperitoneum in the absence of solid organ injury, more than a minimal amount of unexplained peritoneal fluid is a nonspecific but important marker of potentially serious bowel or mesenteric injury. In five major series addressing this issue, the risk of surgically important injury associated with moderate fluid was approximately $50 \%$. Moderate amounts of peritoneal fluid in the presence of a seat belt ecchymosis increases the likelihood of operative intervention to more than $60 \%$, compared to only $6 \%$ in patients with a small amount of fluid.

The advent of multi-detector scanners has improved the accuracy of CT for the diagnosis of bowel and mesenteric injuries. However, reported sensitivity $(80-95 \%)$ and specificity values (48-84\%) range widely among studies. Repeat imaging might improve early identification of bowel injury. In one study of 12 patients with small bowel injury, the frequency of extraluminal air increased from $58 \%$ to $92 \%$ at initial and repeat CT, respectively. Similarly, the frequency of mesenteric fat obliteration $(58 \%$ vs. $75 \%)$ and ascites $(75 \%$ vs. $100 \%)$ also increased after a period of observation.

$\mathrm{CT}$ is an important tool in the early diagnosis of bowel and mesenteric injuries in children. Equally important are a high index of suspicion and serial abdominal examination. Persistent tachycardia, increasing or unremitting abdominal pain in a child with unexplained peritoneal fluid should raise the level of concern about an important missed injury. 